\title{
Éditorial
}

\section{Le tertiaire moteur}

Les spécialistes considèrent généralement que ledit « tertiaire moteur » représente un domaine fondamental dans l'économie des villes. Car il est composé par des activités motrices pour d'autres activités économiques. À titre d'exemple, un plan de bâtiment confectionné par des architectes va générer d'autres activités associées à la construction du bâtiment en planification, notamment l'excavation, les fondations, la charpente, la toiture, l'électricité, la plomberie, etc. Ce domaine du tertiaire moteur de développement économique est généralement circonscrit et défini par les grands champs que voici : la finance, les services spécialisés aux entreprises, la R\&D (recherche et développement), l'enseignement supérieur ainsi que les arts et la culture.

L'analyse urbaine et régionale des activités ou fonctions supérieures dites motrices s'avère très actuelle à une époque de changements technologiques devenus perpétuels par lesquels l'innovation devient la base de la dynamique économique et sociale. La sommité américaine Richard Florida ${ }^{1}$ en a fait le cœur de sa théorie du développement. Il considère les "activités créatrices » comme la force motrice principale de la croissance et du développement des cités, des régions et des nations. Pour ce spécialiste, les champs de cette créativité sont formés de l'ingénierie, de l'enseignement et de la recherche, de la R\&D, des industries technologiques de pointe, des arts et de la culture, de la finance, du droit et de la santé. On constate un rapprochement évident entre ces champs d'activités créatrices de Florida et ceux précités dudit tertiaire moteur classique. Selon Florida, ce vaste domaine d'activités créatrices a plus que doublé son nombre de travailleurs dans les pays industriels avancés au cours de la deuxième moitié du $\mathrm{XX}^{\mathrm{e}}$ siècle. Les villes et les régions, clame cet expert, doivent miser sur leur " classe créatrice ». Dans le contexte très actuel, le tertiaire moteur représente ainsi un objet d'intérêt évident pour la politique publique.

Pour les petites et grandes villes du Québec, le «tertiaire moteur » représente un domaine majeur, névralgique, sur lequel elles doivent miser pour stimuler la créativité, l'innovation et la production. Ces champs du tertiaire moteur au Québec ne peuvent être parfaitement isolés et découpés à partir des statistiques officielles compilées. Parmi les contraintes statistiques à cet effet, signalons que le champ de la finance comprend le secteur des assurances, alors que le champ des arts comprend aussi les loisirs. Néanmoins, un domaine «tertiaire moteur » s'avère identifiable et mesurable sans trop de biais méthodologiques. Sa prise en compte devient fort utile pour comparer, d'une part, les villes entre elles et, d'autre part, les différentes étapes de l'évolution de chaque ville sur une longue période. Il s'agit là de l'exercice analytique que nous effectuons au CRDT (Centre de recherche sur le développement territorial). Plusieurs constats peuvent ainsi être soulevés et servir de base pour une analyse plus poussée. En réalité, l'indice « tertiaire moteur » du CRDT représente une excellente mesure pour saisir et analyser le potentiel futur de polarisation de chaque ville québécoise ${ }^{2}$.

Même si Montréal possède la plus forte masse critique dans le tertiaire moteur au Québec, avec $23 \%$ de l'emploi total dans cette ville, sans compter Laval $(19 \%)$ et Longueuil (23\%), nous avons constaté que la taille de la population n'est pas le seul critère qui influence la position avantageuse de ce moteur de développement. Car Rimouski, à titre d'exemple, possède aussi un tertiaire moteur important qui correspond à $20 \%$ des emplois totaux. Cette position avantageuse s'explique en partie par l'aire de rayonnement de cette capitale régionale de taille limitée. La Malbaie, La Pocatière, Sherbrooke, Saint-Sauveur se positionnent aussi très bien dans leur tertiaire moteur avec des indices respectifs de $19 \%, 21 \%, 19 \%$ et $24 \%$, attribuables en grande partie à leur localisation géographique. Québec $(21 \%)$, Gatineau $(18 \%)$, Lévis (22\%), Trois-Rivières (17\%), Joliette $(16 \%)$, et Saint-Hyacinthe $(17 \%)$ se positionnent relativement bien aussi, pour diverses raisons. Considérant leur propre aire respective de rayonnement très étendue, on peut avancer aussi que les agglomérations de Rouyn (17\%) et de Saguenay (18\%) illustrent un ratio un peu faible en regard de leur potentiel théorique. En outre, des agglomérations telles que Baie-Comeau (12\%), Sept-Îles (13\%), Shawinigan (13\%), Grandby $(12 \%)$, Val d'Or $(12 \%)$ et La Tuque $(8 \%)$ performent faiblement si l'on considère leur rôle de centre de services spécialisés pour un vaste territoire. Par contre, les agglomérations d'Alma (16\%), Carleton (16\%), Nicolet (18\%), Trois-Pistoles (16\%), Maniwaki (15\%), Melbourne (21\%), Mont-Tremblant (20\%) et SaintGeorges $(15 \%)$ et beaucoup d'autres centres urbains secondaires et même tertiaires possèdent, selon notre indice, un domaine du tertiaire moteur relativement dynamique et porteur de développement. 
Notre indice comparatif illustre bien sûr des inégalités interterritoriales dans la dotation en activités tertiaires motrices de développement. On constate aussi une déconcentration assez importante de ces activités supérieures sur le vaste espace du Québec. De très nombreuses petites villes périphériques comme Saint-Félicien, Matane, Beauceville possèdent ainsi les ressorts adéquats pour générer la créativité, l'innovation et le développement économique et social sur leur territoire immédiat et éloigné. Ces ressorts s'inscrivent concrètement dans leurs activités reliées à l'éducation, la finance, les services privés et publics aux entreprises, les arts et la culture, la R\&D.

Bel et bien présentes à degrés divers dans les villes québécoises, les activités du secteur tertiaire moteur s'avèrent non seulement vulnérables, mais confrontées actuellement dans leur survie. L'intégration des activités par les grands groupes est un phénomène réel, dans un contexte de très forte concurrence. Ce qui favorise la desserte de services à partir des grands centres urbains comme Montréal et Toronto. Aussi, la centralisation décisionnelle des entreprises et des agences gouvernementales localisées en régions ne favorise guère le tertiaire moteur des petites et moyennes villes. En outre, les grandes entreprises aux unités déconcentrées, notamment dans les secteurs des pâtes et papier, de l'aluminium, des mines et du bois d'œuvre, appliquent trop souvent la stratégie de réduction du nombre de fournisseurs de services. Bref, le domaine du tertiaire moteur est malmené dans les petites et moyennes villes du Québec. Certaines activités sont évidemment plus touchées que d'autres. Mais, en général, ces petits noyaux moteurs de développement local et régional sont actuellement fragilisés.

De façon générale, on peut supposer qu'au cours des prochaines années, les activités qui composent le domaine du tertiaire moteur dans les petites et moyennes villes devront s'orienter vers la diversification de leurs services, alors que certains clients traditionnels seront de moins en moins présents dans leur économie locale et régionale. Ces activités devront miser davantage sur la PME spécialisée dans des niches qui demandera des services très spécialisés. Elles devront faire preuve de créativité, de flexibilité, d'adaptation et d'innovation en se collant aux besoins du client souvent peu révélés, mais à détecter impérativement et à satisfaire adéquatement pour l'assister à demeurer concurrentiel.

Terminons l'éditorial de ce premier numéro de 2005 en soulignant que le domaine " tertiaire moteur » possède un potentiel important dans les petites et moyennes villes périphériques du Québec. D'abord, parce qu'en ces lieux non métropolitains, la structure des coûts de production de services spécialisés s'avère généralement avantageuse. Certaines firmes spécialisées ont même récemment déconcentré des unités de production hors des grands centres urbains afin de maximiser leur compétitivité. Ensuite, parce que la présence de services publics supérieurs au sein de nombreuses villes (collèges, centres de R\&D, incubateurs, hôpitaux, centres de formation professionnelle, universités...) offre une masse critique de compétences et un noyau entraînant d'expertise qui soutiennent par essence le développement du tertiaire moteur.

Aussi, la mise en œuvre ponctuelle de "grands travaux » tels que la construction de barrages, d'usines, de routes, de ports, de bâtiments importants et autres équipements et infrastructures, notamment dans le nord du Québec, génère non seulement de l'ouvrage mais aussi des avancées dans les expertises pointues. L'entretien de ces équipements et ces infrastructures devient une activité professionnelle de plus en plus importante.

Finalement, de nouveaux créneaux et niches émergent constamment sur le vaste territoire du Québec, en créant une demande importante de services spécialisés. Nous pensons tout de suite aux niches de la $2^{\mathrm{e}}$ et de la $3^{\mathrm{e}}$ transformation des matières premières, notamment dans le bois. Soulignons aussi l'aménagement forestier dont le vaste chantier déjà amorcé représente un champ qui nécessite beaucoup d'expertise et d'innovation. Et que dire des autres créneaux en développement tels que les technomines, l'hydroélectricité, l'aluminium, les populations autochtones, l'éolien, l'agriculture nordique, le récréotourisme, la défense, etc. qui nécessitent beaucoup d'expertise issue du domaine professionnel qu'on appelle le tertiaire moteur?

Bref, au Québec, le domaine du tertiaire moteur établi dans les villes de diverses tailles localisées en divers endroits bénéficie de solides acquis, possède un potentiel considérable mais illustre actuellement une fragilité évidente qui menace sa pérennité. Il va sans dire que des mesures publiques spécifiquement ciblées pour soutenir adéquatement ses activités spécifiques, selon les lieux et les milieux, seraient souhaitables, notamment là où les effets de polarisation illustrent des chances de devenir entraînants pour le bénéfice de ces villes et des territoires environnants.

\section{Marc-Vrbain Proulx}

\section{Le Directeur}

\section{Notes et références}

1 Florida, Richard (2005). "Cities and the Creative Class », New York, Routledge Publications, 198 p.

2 Nous utilisons les découpages politiques et administratifs définis par la réforme des agglomérations urbaines de 2002. 